\title{
Impacto e manejo obstétrico de pacientes grávidas infectadas pelo SARS-CoV-2
}

\author{
Impact and obstetric management of pregnant patients infected with SARS-CoV-2 \\ Impacto y manejo obstétrico de pacientes embarazadas infectadas por SARS-CoV-2
}

Recebido: 17/12/2021 | Revisado: 24/12/2021 | Aceito: 29/12/2021 | Publicado: 06/01/2022

Ana Klara Rodrigues Alves

ORCID: https://orcid.org/0000-0002-1216-9386 Universidade Estadual do Piauí, Brasil E-mail:klaraphb@outlook.com

Barbara Beatriz Lira da Silva

ORCID: https://orcid.org/0000-0001-9166-6147 Universidade Estadual do Piauí, Brasil

E-mail: brbeatriz16@gmail.com

Lívia Filomena Castelo Branco Machado

ORCID: https://orcid.org/0000-0001-5073-8140

Centro Universitário UNINOVAFAPI, Brasil

E-mail: liviafilomenacbm@hotmail.com

Gabriel Brito da Silva

ORCID: https://orcid.org/0000-0002-9765-1074

Faculdade de Ciências Humanas, Exatas e da Saúde do Piauí, Brasil

E-mail: bielgnr@hotmail.com

José Vieira Amorim Filho

ORCID: https://orcid.org/0000-0002-4929-8253

Faculdade de Ciências Humanas, Exatas e da Saúde do Piaú, Brasil

E-mail: vieirajose330@gmail.com

Francisco das Chagas da Silva Serejo Júnior

ORCID: https://orcid.org/0000-0002-5167-2830

Faculdade de Ciências Humanas, Exatas e da Saúde do Piaú, Brasil

E-mail: fserejojunior@hotmail.com

Mylenna Maria de Brito Silva

ORCID: https://orcid.org/0000-0002-5701-6404

Centro Universitário UNINOVAFAPI, Brasil

E-mail: mylennambs@gmail.com

Amanda Milhomem Medeiros

ORCID: https://orcid.org/0000-0002-3953-0442

Faculdade de Ciências Humanas, Exatas e da Saúde do Piaú, Brasil

E-mail: amanda_medeiros07@hotmail.com

Yama Vitoriano Lima

ORCID: https://orcid.org/0000-0002-0802-4221

Faculdade de Ciências Humanas, Exatas e da Saúde do Piauí, Brasil

E-mail: yamavitoriano@hotmail.com

Raissa Martins de Oliveira Nunes

ORCID: https://orcid.org/0000-0002-4741-2489

Faculdade de Ciências Humanas, Exatas e da Saúde do Piauí, Brasil

E-mail: raiissamartiins@ hotmail.com

Victor Rocha Santos

ORCID: https://orcid.org/0000-0002-8068-3264

Centro Universitário UNINOVAFAPI, Brasil

E-mail: victoroxas@gmail.com

Verbena Krieger Rocha Santos

ORCID: https://orcid.org/0000-0002-5901-2255

Universidade federal do Maranhão, Brasil

E-mail: verbenakrieger@gmail.com

Danielle Sotero Fortes Carvalho

ORCID: https://orcid.org/0000-0002-1563-2946

Centro Universitário UNINOVAFAPI, Brasil

E-mail: dani_fortes1995@hotmail.com

Maria Clara Martins Costa

ORCID: https://orcid.org/0000-0002-6264-4069

Centro Universitário UNINOVAFAPI, Brasil

E-mail: myrianclara@hotmail.com

Iluska Guimarães Rodrigues

ORCID: https://orcid.org/0000-0003-2966-9018

Centro Universitário UNINOVAFAPI, Brasil

E-mail: iluska.guimaraes@gmail.com 


\title{
Resumo
}

Podem ocorrer alterações fisiológicas e imunológicas maternas durante a gravidez, as mulheres grávidas podem correr um risco maior de serem infectadas com SARS-CoV-2 e desenvolver eventos clínicos mais complicados. O presente estudo tem como objetivo descrever o impacto do covid-19 durante a gravidez e resumir os possíveis sintomas, tratamentos e resultados da gravidez de mulheres infectadas com Covid-19 durante a gravidez. Realizado através da Biblioteca Virtual de Saúde (BVS) nas bases de dados do Pubmed e Scielo, usando os cruzamentos dos descritores em inglês "Pregnancy",“Covid-19", "Complications". Para a avaliação do problema de pesquisa e sua estratificação foi utilizada a estratégia PVO. A estratégia supracitada permitiu formular a seguinte questão norteadora: Quais são os impactos e complicações causadas pelo covid-19 durante a gestação? A partir da questão norteadora foram utilizados os operadores booleanos para a sistematização das buscas com o seguinte esquema: Pregnancy AND Covid-19 AND complications. Algumas complicações na gravidez ocorreram em mulheres grávidas com Covid-19, como sofrimento fetal, ruptura prematura de membranas, partos prematuros e natimortos. Além disso, essas complicações na gravidez podem estar intimamente relacionadas à tempestade de citocinas, lesão pulmonar e isquemia / hipóxia placentária causada por infecções por SARS-CoV-2. O aumento da vulnerabilidade das mulheres grávidas bem como a experiência anterior de epidemias de coronavírus anteriores é motivo de preocupação para complicações maternas e fetais. O rápido surto da doença, combinado com a incerteza, como resultado direto da falta de dados científicos sólidos, tem forçado os obstetras a adaptarem suas práticas atuais de forma pragmática.

Palavras-chave: Covid-19; Gravidez; Complicações.

\begin{abstract}
Maternal physiological and immunological changes can occur during pregnancy, pregnant women may be at increased risk of being infected with SARS-CoV-2 and develop more complicated clinical events. The present study aims to describe the impact of covid-19 during pregnancy and summarize the possible symptoms, treatments, and pregnancy outcomes of women infected with Covid-19 during pregnancy. Carried out through the Virtual Health Library (VHL) in the Pubmed and Scielo databases, using the crossings of the descriptors in English "Pregnancy", "Covid-19", "Complications". To evaluate the research problem and its stratification, the PVO strategy was used. The aforementioned strategy allowed the formulation of the following guiding question: What are the impacts and complications caused by covid-19 during pregnancy? From the guiding question, Boolean operators were used to systematize the searches with the following scheme: Pregnancy AND Covid-19 AND complications. Some pregnancy complications have occurred in pregnant women with Covid-19, such as fetal distress, premature rupture of membranes, premature births and stillbirths. Furthermore, these pregnancy complications may be closely related to cytokine storm, lung injury, and placental ischemia/hypoxia caused by SARS-CoV-2 infections. The increased vulnerability of pregnant women as well as the past experience of previous coronavirus outbreaks is of concern for maternal and fetal complications. The rapid outbreak of the disease, combined with uncertainty as a direct result of the lack of solid scientific data, has forced obstetricians to adapt their current practices in a pragmatic way.
\end{abstract}

Keywords: Covid-19; Pregnancy; Complications.

\section{Resumen}

Los cambios fisiológicos e inmunológicos maternos pueden ocurrir durante el embarazo, las mujeres embarazadas pueden tener un mayor riesgo de infectarse con el SARS-CoV-2 y desarrollar eventos clínicos más complicados. El presente estudio tiene como objetivo describir el impacto del Covid-19 durante el embarazo y resumir los posibles síntomas, tratamientos y resultados del embarazo de las mujeres infectadas con Covid-19 durante el embarazo. Realizado a través de la Biblioteca Virtual en Salud (BVS) en las bases de datos Pubmed y Scielo, utilizando los cruces de los descriptores en inglés "Pregnancy", "Covid-19", "Complications". Para evaluar el problema de investigación y su estratificación se utilizó la estrategia PVO. La estrategia antes mencionada permitió formular la siguiente pregunta orientadora: ¿Cuáles son los impactos y complicaciones que provoca el covid-19 durante el embarazo? A partir de la pregunta orientadora, se utilizaron operadores booleanos para sistematizar las búsquedas con el siguiente esquema: Embarazo Y Covid-19 Y complicaciones. Algunas complicaciones del embarazo han ocurrido en mujeres embarazadas con Covid-19, como sufrimiento fetal, ruptura prematura de membranas, partos prematuros y mortinatos. Además, estas complicaciones del embarazo pueden estar estrechamente relacionadas con la tormenta de citocinas, la lesión pulmonar y la isquemia / hipoxia placentaria causadas por infecciones por SARS-CoV-2. La mayor vulnerabilidad de las mujeres embarazadas, así como la experiencia pasada de brotes de coronavirus anteriores, es motivo de preocupación por las complicaciones maternas y fetales. El rápido brote de la enfermedad, combinado con la incertidumbre como resultado directo de la falta de datos científicos sólidos, ha obligado a los obstetras a adaptar sus prácticas actuales de manera pragmática.

Palabras clave: Covid-19; Embarazo; Complicaciones.

\section{Introdução}

A pandemia causada pela síndrome respiratória aguda grave coronavírus 2 (SARS-CoV-2), agente etiológico da 
Covid-19, tem se propagado no mundo inteiro de maneira rápida, vulnerabilizando, dentre outros grupos, as gestantes e seus fetos. Diante das complicações para a gestação e o feto, faz-se necessário refletir sobre o estar gestante em tempos de pandemia da Covid-19 e a importância do cuidado profissional, a fim de superar os inúmeros desafios que permeiam esse contexto (Wang et al.,2021).

Podem ocorrer alterações fisiológicas e imunológicas maternas durante a gravidez, as mulheres grávidas podem correr um risco maior de serem infectadas com SARS-CoV-2 e desenvolver eventos clínicos mais complicados. Informações sobre a síndrome respiratória aguda grave (SARS) e a síndrome respiratória do Oriente Médio (MERS) podem fornecer informações sobre os efeitos do Covid-19 durante a gravidez. Embora SARS e MERS tenham sido associados a aborto espontâneo, morte intrauterina, restrição de crescimento fetal e altas taxas de letalidade, o curso clínico da pneumonia por Covid-19 em mulheres grávidas foi relatado como semelhante ao de mulheres não grávidas. Além disso, as mulheres grávidas não parecem ter um risco maior de contrair Covid-19 ou de sofrer de doença mais grave do que outros adultos da mesma idade, na maioria dos infectados, os sintomas apresentados são leves, a exemplo de febre e tosse seca, porém, em mulheres na segunda metade da gestação, há outros sintomas que podem aparecer com menor intensidade nas gestantes, como fadiga, dispneia, diarreia, congestão nasal e coriza. Algumas mulheres podem apresentar ainda complicações mais graves, como a síndrome respiratória aguda grave (SARS) (Wenling et al., 2020).

Mudanças fisiológicas e mecânicas na gravidez aumentam a suscetibilidade a infecções em geral, particularmente quando o sistema cardiorrespiratório é afetado, e encorajam a rápida progressão para insuficiência respiratória na gestante. Além disso, o viés da gravidez para a dominância do sistema T-helper 2 (Th2), que protege o feto, deixa a mãe vulnerável a infecções virais, que são contidos de forma mais eficaz pelo sistema T-helper 1 (Th1). Esses desafios únicos exigem uma abordagem integrada para gestações afetadas pela SARS-CoV-2 (Dashraath et al., 2020).

Yang et al. (2020) enfatiza que as mulheres grávidas geralmente estão em um grupo de alto risco para doenças infecciosas devido às alterações imunológicas e fisiológicas gestacionais em seu sistema. De acordo com experiências anteriores com SARS, MERS e influenza, as mulheres grávidas são mais propensas a desenvolver pneumonia grave em caso de infecção por patógenos respiratórios do que pacientes não grávidas.

O presente estudo tem como objetivo descrever o impacto do Covid-19 durante a gravidez e resumir os possíveis sintomas, tratamentos e resultados da gravidez de mulheres infectadas com Covid-19 durante a gravidez.

\section{Metodologia}

Trata-se de um estudo de abordagem qualitativa, onde se adotou a revisão integrativa da literatura, que conforme Galvão (2012), é uma construção de uma análise ampla da literatura com passos pré-definidos uma vez que ela contribui para o processo de sistematização e análise dos resultados, visando a compreensão de determinado tema, a partir de outros estudos independentes. Realizado através da Biblioteca Virtual de Saúde (BVS) nas bases de dados do Pubmed e Scielo, usando os cruzamentos dos descritores em inglês "Pregnancy", "Covid-19", "Complications". Para a avaliação do problema de pesquisa e sua estratificação foi utilizada a estratégia PVO (População/ Problema, Variável/Resultados e Outcomes/ Desfechos) sendo formulada a seguinte estratégia que pode ser observada no Quadro 1. A estratégia supracitada permitiu formular a seguinte questão norteadora: Quais são os impactos e complicações causadas pelo Covid-19 durante a gestação? A partir da questão norteadora foram utilizados os operadores booleanos para a sistematização das buscas com o seguinte esquema: Pregnancy AND Covid-19 AND complications. 
Quadro 1. Estratificação do problema de pesquisa seguindo estratégia PVO para formulação de pesquisa.

\begin{tabular}{|c|l|}
\hline $\begin{array}{c}\text { P } \\
\text { População }\end{array}$ & Gestantes \\
\hline $\begin{array}{c}\text { V } \\
\text { Variáveis }\end{array}$ & Covid-19 e complicações que ocorrem durante da gestação \\
\hline $\begin{array}{c}\text { O } \\
\text { Desfechos }\end{array}$ & Verificar quais as complicações causadas pelo Covid-19 na gestação. \\
\hline
\end{tabular}

Fonte: Autores (2021).

Para a seleção dos artigos foram utilizados os seguintes critérios de inclusão: artigos escritos na língua inglesa e portuguesa; publicados entre os anos 2020 até o segundo semestre de 2021 e que abordem sobre o impacto e as complicações causadas pelo covid-19 na gestação. No que diz respeito aos critérios de exclusão, dispensaram-se artigos que se distanciavam da temática central desta revisão e trabalhos que não apresentassem resumos na íntegra nas bases de dados pesquisadas. Após a pré-leitura e leitura seletiva dos textos, foram selecionados 21 artigos (Quadro 2), nos quais realizou-se uma leitura interpretativa buscando responder à pergunta de pesquisa desta revisão.

\section{Resultados e Discussão}

A partir das buscas realizadas nas bases de dados, foi encontrado um total de 2.010 artigos, sendo selecionados 20 artigos, destes 20 foram encontrados na base de dados Pubmed e 1 na Scielo.

Quadro 2. Distribuição dos artigos segundo o título, autores, objetivo, abordagem do artigo, revista e ano.

\begin{tabular}{|c|c|c|c|c|c|}
\hline TITULO & AUTORES & OBJETIVO & ABORDAGEM & REVISTA & $\begin{array}{c}\text { ANO DE } \\
\text { PUBLICAÇÃO }\end{array}$ \\
\hline $\begin{array}{l}\text { Impact of COVID-19 } \\
\text { on Pregnancy }\end{array}$ & $\begin{array}{l}\text { Wang, CL, Liu, YY, } \\
\text { Wu, CH, Wang, CY, } \\
\text { Wang, CH, \& Long, } \\
\text { CY }\end{array}$ & $\begin{array}{l}\text { Resumir os possíveis sintomas, tratamentos e } \\
\text { resultados da gravidez de mulheres infectadas com } \\
\text { COVID-19 durante a gravidez. }\end{array}$ & Manejo clínico & $\begin{array}{l}\text { Jornal internacional } \\
\text { de ciências médicas }\end{array}$ & 2021 \\
\hline $\begin{array}{l}\text { Pregnancy } \quad \text { and } \\
\text { COVID-19: } \\
\text { management } \\
\text { challenges }\end{array}$ & $\begin{array}{l}\text { Wenling, Y., } \\
\text { Junchao, Q., Xiao, } \\
\text { Z., \& Ouyang, S. }\end{array}$ & $\begin{array}{l}\text { Fornece uma introdução à patogênese, patologia e } \\
\text { características clínicas do COVID-19 e enfocou as } \\
\text { pesquisas atuais sobre características clínicas, } \\
\text { resultados da gravidez e análise histopatológica } \\
\text { placentária de mulheres grávidas infectadas com } \\
\text { SARS -CoV-2 em comparação com SARS-CoV e } \\
\text { MERS-CoV }\end{array}$ & Fisiopatologia & $\begin{array}{l}\text { Revista do Instituto } \\
\text { de Medicina } \\
\text { Tropical de São } \\
\text { Paulo }\end{array}$ & 2020 \\
\hline $\begin{array}{l}\text { "Coronavirus Disease } \\
2019 \quad \text { (COVID-19) } \\
\text { Vaccines and } \\
\text { Pregnancy: What } \\
\text { Obstetricians } \\
\text { Know" Need to }\end{array}$ & $\begin{array}{l}\text { Rasmussen, S. A., } \\
\text { Kelley, C. } \\
\text { Horton, J. P., \& } \\
\text { Jamieson, D. J. }\end{array}$ & $\begin{array}{l}\text { Resumir o que é conhecido atualmente sobre as } \\
\text { vacinas COVID-19 e seu uso durante a gravidez. }\end{array}$ & Fisiopatologia & $\begin{array}{l}\text { Obstetrics and } \\
\text { gynecology }\end{array}$ & 2021 \\
\hline $\begin{array}{l}\text { COVID-19 and } \\
\text { pregnancy }\end{array}$ & $\begin{array}{l}\text { Masmejan S, Pomar } \\
\text { L, Lepigeon K, } \\
\text { Favre G, Baud D, } \\
\text { Rieder W. }\end{array}$ & $\begin{array}{l}\text { Revisar o manejo obstétrico de pacientes grávidas } \\
\text { infectadas pelo SARS-CoV-2 com base no } \\
\text { conhecimento atual. }\end{array}$ & Manejo clínico & Rev Med Suisse. & 2020 \\
\hline $\begin{array}{lr}\text { COVID-19 } & \text { vaccines } \\
\text { and } & \text { neglected } \\
\text { pregnancy } & \end{array}$ & $\begin{array}{l}\text { Dashraath, } \\
\text { Nielsen-Saines, K., } \\
\text { Madhi, S. A., \& } \\
\text { Baud, D. }\end{array}$ & $\begin{array}{l}\text { Os protocolos devem incluir disposições para } \\
\text { monitoramento da segurança materna e fetal e para } \\
\text { documentação de complicações iatrogênicas, } \\
\text { incluindo acompanhamento da prole após o parto. }\end{array}$ & Protocolo clínico & The Lancet & 2020 \\
\hline $\begin{array}{l}\text { Characteristics of } \\
\text { Women of } \\
\text { Reproductive Age } \\
\text { with Laboratory- } \\
\text { Confirmed SARS- } \\
\text { CoV-2 Infection by } \\
\text { Pregnancy Status }\end{array}$ & 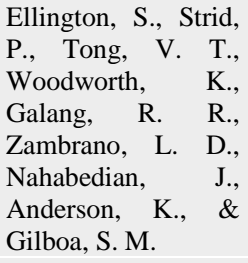 & $\begin{array}{l}\text { Descrever as características de mulheres em idade } \\
\text { reprodutiva com infecção por SARS-CoV-2 } \\
\text { confirmada em laboratório por estado de gravidez. }\end{array}$ & Fisiopatologia & $\begin{array}{l}\text { Morbidity and } \\
\text { mortality weekly } \\
\text { report }\end{array}$ & 2020 \\
\hline $\begin{array}{l}\text { Universal Screening } \\
\text { for SARS-CoV-2 in } \\
\text { Women Admitted for } \\
\text { Delivery }\end{array}$ & $\begin{array}{l}\text { Sutton, D., Fuchs, } \\
\text { K., D'Alton, M., \& } \\
\text { Goffman, D. }\end{array}$ & $\begin{array}{l}\text { Realizar a triagem universal para SARS-CoV-2 em } \\
\text { mulheres admitidas para parto }\end{array}$ & Manejo clínico & $\begin{array}{l}\text { The New England } \\
\text { journal of medicine }\end{array}$ & 2020 \\
\hline
\end{tabular}




\begin{abstract}
Clinical
characteristics and intrauterine vertical transmission potential of COVID-19 infection in nine pregnant women: a retrospective review of medical records.

Clinical predictors of mortality due to COVID-19 based on an analysis of data of 150 patients from Wuhan, China
\end{abstract}

Clinical features of patients infected with 2019 novel coronavirus in Wuhan China.

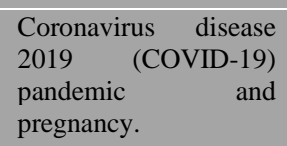

pregnancy.

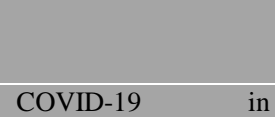

pregnancy

COVID-19

pregnancy: Placental

and neonatal

involvement.

COVID-19 and

pregnancy - where are

we now? A review.

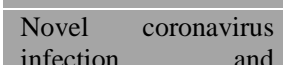

infection and

pregnancy

\section{Pregnant and} postpartum women with severe acute respiratory syndrome coronavirus

infection in intensive care in Sweden.

Pregnancy

COVID-19:

pharmacologic

considerations.
Chen, H., Guo, J., Wang, C., Luo, F., Yu, X., Zhang, W., Li, J., Zhao, D., Xu, D., Gong, Q., Liao, J., Yang, H., Hou, W., \& Zhang, Y.

Ruan, Q., Yang, K., Relatar pela primeira vez que a infecção de SARS-

Wang, W., Jiang, L., CoV-2 pode causar miocardite fulminante. Dado que \& Song, J.

Huang, C., Wang, Y., Li, X., Ren, L., Zhao, J., Hu, Y., Zhang, L., Fan, G., $\mathrm{Xu}$, J., Gu, X., Cheng, Z., Yu, T., Xia, J., Wei, Y., Wu, W., Xie, X., Yin, W., Li, H., Liu, M., Xiao, Y., ... Cao, B.

Dashraath, P., Wong, J., Lim, M., Lim, L. M., Li, S., Biswas, A., Choolani, M.,

Mattar, C., \& Su, L. L. (2020).

J.G. Thorton

Prochaska, E., Jang, M., \& Burd, I.

Aleksandra

Rajewska, Wioletta Mikołajek-Bedner,

Joanna Lebdowicz-

Knul, Małgorzata

Sokołowska,

Sebastian

Kwiatkowski and

Andrzej Torbé

H. Yang,C. Wang,L.

C. Poon

Collin, J., Byström,

E., Carnahan, A., \& Ahrne, $\mathrm{M}$.

D'Souza, R., Ashraf, R., Rowe, H., Zipursky, Clarfield, Maxwell, Arzola,

Lapinsky,

Paquette,

Murthy, S., Cheng,

M. P., \& Malhamé,

I.

Avaliar as características clínicas de COVID-19 na gravidez e o potencial de transmissão vertical intrauterina da infecção por COVID-19. a miocardite fulminante é caracterizada por uma evolução rápida e um estado de doença grave [ 3 ], nossos resultados devem alertar os médicos a prestar atenção não apenas aos sintomas de disfunção respiratória, mas também aos sintomas de lesão cardíaca.

Relatar as características epidemiológicas, clínicas, laboratoriais e radiológicas e o tratamento e resultados clínicos desses pacientes.

Compartilhar uma estrutura que pode ser adotada por maternidades terciárias que gerenciam mulheres grávidas no fluxo de uma pandemia, mantendo a segurança do paciente e do profissional de saúde em seu núcleo.

Esclarecer as manifestações clínicas que ocorrem na gestação.

Resumir a literatura conhecida sobre a placenta na infecção por SARS - CoV - 2, evidências de transmissão vertical e possíveis resultados de exposição pré-natal ao vírus.

Entender sobre as manifestações clínicas e alterações que podem ocorrer no organismo das gestantes que estão infectadas por covid-19

Revisamos a manifestação clínica, a evolução neonatal e o risco de transmissão vertical da infecção por COVID-19 durante a gravidez.

Realizar o acompanhamento de Mulheres grávidas e puérperas com infecção por SARS - CoV - 2 em terapia intensiva na Suécia

Avaliamos criticamente a orientação em relação às considerações farmacológicas exclusivas para
mulheres grávidas e lactantes com doença por coronavírus (COVID-19).
Fisiopatologia

The Lancet

2020

Fisiopatologia

Intensive care

2020

medicine

Fisiopatologia e

The Lancet

Protocolo clínico

American journal of obstetrics and

gynecology.

Fisiopatologia

BJOG: An

Fisiopatologia

International Journa

of Obstetrics \&

Gynaecology

American journal of reproductive immunology

Fisiopatologia

Journal of Perinatal Medicine

Manejo clínico

BJOG: An

International Journal of Obstetrics \&

Gynaecology

Manejo clínico

Acta Obstet Gynecol Scand.

Protocolo clínico

Ultrasound in

Obstetrics and 


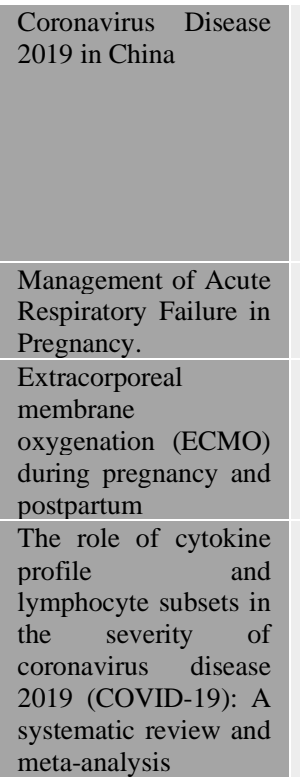

Yu Hu, Wen-hua Liang, Chun-quan $\mathrm{Ou}$, Jian-xing $\mathrm{He}$,

Lei Liu, Hong Shan,Chun-liang Lei, David S.C. Hui, Bin Du, Lan-juan $\mathrm{Li}$, et al.

Lapinsky SE.

Pacheco LD, Saade GR, Hankins GDV.

Akbari, H., Tabrizi, R., Lankarani, K. B., Aria, H., Vakili, S., Asadian, $\quad$ F., Noroozi, S., Keshavarz, P., \& Faramarz, $S$. resultados de nossa análise das características clínicas de Covid-19 em uma coorte selecionada de pacientes em toda a China. algumas diferenças no manejo da gestante com insuficiência respiratória aguda.

Utilização da oxigenação por membrana extracorpórea (ECMO) durante a gravidez e pósparto

Fazer uma comparação entre os fatores relacionados ao laboratório clínico, índices de hemograma completo (CBC), citocinas e subconjuntos de linfócitos, a fim de distinguir os casos de doença coronavírus grave 2019 (COVID-19) daqueles não graves.

\section{Protocolo clínico Semin Respir Crit Care Med.}

Protocolo clínico Semin Perinatol 2020

Fonte: Autores (2021).

Existe um crescente corpo de evidências que sugere que as mulheres grávidas correm um maior risco de morbidade e mortalidade por Covid-19, incluindo maior risco de insuficiência respiratória com a necessidade de admissão em cuidados intensivos e ventilação mecânica, em comparação com mulheres da mesma idade que não estão grávidas. Covid-19 também foi associado a um aumento da taxa de natimortos (Dashraath et al., 2020).

$\mathrm{Na}$ maioria das mulheres grávidas o curso da doença foi leve, o que é definido como pneumonia não pneumônica ou leve. Mulheres grávidas com pneumonia por Covid-19 mostraram um padrão semelhante em comparação com mulheres não grávidas, incluindo febre, tosse, mialgia, fadiga, falta de ar ou apresentação assintomática. Os casos graves foram caracterizados por dispneia, taquipneia, diminuição da saturação de oxigênio no sangue, queda da razão da pressão parcial de oxigênio arterial para a fração de oxigênio inspirado e / ou infiltração pulmonar maciça. Comorbidades pré-existentes como doenças cardiovasculares, diabetes, doenças respiratórias crônicas, hipertensão e câncer aumentaram significativamente a taxa geral de letalidade (Rajewska et al., 2020; Ellington et al., 2020).

Algumas complicações na gravidez ocorreram em mulheres grávidas com Covid-19, como sofrimento fetal, ruptura prematura de membranas, partos prematuros e natimortos. Além disso, essas complicações na gravidez podem estar intimamente relacionadas à tempestade de citocinas, lesão pulmonar e isquemia / hipóxia placentária causada por infecções por SARS-CoV-2 (Wenling et al., 2020).

As citocinas produzidas por linfócitos $\mathrm{T}$ auxiliares (Th) regulam a imunidade e a inflamação. As citocinas do tipo Th1 são microbicidas e pró-inflamatórias, em contraste, as citocinas do tipo Th2 são anti-inflamatórias. Na gravidez, a atenuação da imunidade mediada por células por células Th1 devido à mudança fisiológica para um ambiente dominante Th2 contribui para a morbidade infecciosa geral, aumentando a suscetibilidade materna a patógenos intracelulares, como vírus (Huang et al., 2020; Ruan et al., 2020).

A enzima conversora de angiotensina 2 (ACE2) foi identificada como o receptor funcional para SARS-CoV-2. O fato de ACE2 estar abundantemente presente no epitélio dos pulmões e do intestino delgado, fornece uma possível explicação para os sintomas patológicos do pulmão e gastrointestinais. A expressão abundante de ACE2 nas células alveolares pode causar uma rápida expansão viral e destruição da parede alveolar, resultando em uma rápida progressão de consolidações pulmonares extensas e dano alveolar difuso com formação de membrana hialina. Sua presença no endotélio vascular também pode fornecer 
um passo adiante no entendimento das alterações histológicas das placentas de mulheres grávidas infectadas pelo SARS-CoV2 (Wan et al., 2020).

O diagnóstico é baseado na história de exposição, manifestação clínica e resultado de exames laboratoriais. Um ensaio em tempo real da reação em cadeia da polimerase da transcriptase reversa (RT-PCR) é o padrão ouro para o diagnóstico. A radiografia de tórax (CXR) e a tomografia computadorizada (TC) de tórax podem auxiliar no diagnóstico e podem ser usadas para avaliar a extensão e o acompanhamento do Covid-19. A radiografia torácica pode ser realizada de forma rápida e fácil à beira do leito, enquanto a TC de tórax é mais sensível no estágio inicial da infecção. No entanto, as preocupações com relação aos potenciais efeitos teratogênicos da exposição à radiação para o feto são inevitáveis (Masmejan et al., 2020; Wang et al., 2021).

Considerando que a hospitalização ocorreu em uma proporção significativamente maior de mulheres grávidas do que mulheres não grávidas. A admissão na UTI e o recebimento de ventilação mecânica são proxies distintos para a gravidade da doença, e depois de ajustar para idade, presença de doenças subjacentes e raça / etnia, os riscos para ambos os resultados foram significativamente maiores entre mulheres grávidas do que entre mulheres não grávidas (Sutton, Fuchs, D’Alton \& Goffman, 2020). Esses achados são semelhantes aos de um estudo recente na Suécia, que constatou que mulheres grávidas com Covid-19 tinham cinco vezes mais chances de serem admitidas em UTI e quatro vezes mais de receber ventilação mecânica do que mulheres não grávidas. O risco de morte foi o mesmo para mulheres grávidas e não grávidas (Collin et al., 2020).

Mudanças nos sistemas cardiorrespiratório e imunológico durante a gravidez aumentam a suscetibilidade da mulher a infecções graves e comprometimento da hipóxia, mas também podem atrasar o diagnóstico e o controle da fonte naquelas com apenas sintomas inócuos do trato respiratório superior, como dor de garganta e congestão nasal. A rinite gestacional, devido à hiperemia da nasofaringe mediada por estrogênio, geralmente afeta um quinto das mulheres saudáveis no final da gravidez e resulta em congestão nasal acentuada e rinorreia; essas características podem mascarar os sintomas corizais de Covid-19, levando a uma disseminação viral não controlada e à transmissão na comunidade (Guan et al., 2020).

Modificação no padrão de frequência cardíaca fetal pode ser um indicador precoce de deterioração respiratória materna. Com base na experiência com SARS e MERS, pode ocorrer insuficiência respiratória grave em mulheres grávidas e, nos casos mais graves, a ventilação mecânica pode não ser suficiente para suportar a oxigenação adequada. Se isso ocorrer, a literatura limitada sugere um papel potencial da oxigenação por membrana extracorpórea na gravidez; o uso deve ser considerado apenas em centros com experiência com essa técnica. Não se sabe se o parto traz benefícios para uma mãe gravemente doente; as decisões relacionadas ao parto devem levar em consideração a idade gestacional do feto e devem ser feitas em conjunto com o neonatologista (Pacheco et al., 2018; Lapinsky, 2017).

Como acontece com todas as decisões relacionadas ao tratamento durante a gravidez, é necessário avaliar cuidadosamente os benefícios das intervenções para a mãe e o feto com os riscos potenciais. Não há tratamento específico para a infecção por SARS-CoV-2, os procedimentos típicos incluem oxigenoterapia, antibióticos, agentes antiinflamatórios e terapia antiviral. Recomenda-se realizar o manejo em uma unidade de saúde com monitoramento materno e fetal rigoroso, incluindo isolamento precoce, procedimentos agressivos de controle de infecção, oxigenoterapia, prevenção de sobrecarga de fluidos, antibióticos empíricos para possível superinfecção bacteriana, SARS-CoV-2 e teste de coinfecção, controle da atividade fetal e uterina, implementação precoce da ventilação mecânica se necessário, planejamento do modo de parto individual e consultas com várias especialidades, se necessário (Yang et al., 2020; Sutton et al., 2020).

D'Souza et al. (2020) apoia o uso rotineiro de corticosteroides pré-natais, sulfato de magnésio e aspirina em baixa dosagem, quando clinicamente indicado, e se não for contra-indicado por razões médicas. Ressalta que a tomada de decisão sobre o início, dose e duração da anticoagulação profilática para pacientes grávidas com Covid-19 deve ser feita por uma 
equipe multidisciplinar e deve considerar a gravidade da doença, momento do parto em relação ao início da doença, situação do paciente internado versus ambulatorial, comorbidades subjacentes e contra-indicações ao uso de anticoagulação.

A maioria das mulheres grávidas foi submetida a parto cesáreo, pois eram sintomáticas com pneumonia Covid-19 no terceiro trimestre da gravidez. O intervalo de tempo desde a manifestação clínica da infecção por Covid-19 até o parto cesáreo foi curto (variação de 1 a 7 dias). Portanto, é incerto se há risco de transmissão vertical se o intervalo entre a manifestação clínica e o parto for superior a 7 dias. É recomendado permitir o parto vaginal apenas em caso de início espontâneo do trabalho de parto, com um segundo estágio encurtado do trabalho de parto (Thornton, 2020).

Relatos de casos indicam que a transmissão vertical é incomum; no entanto, há evidências de que pode ocorrer infecção placentária e fetal. Placentas de pacientes infectados mostram alterações inflamatórias, trombóticas e vasculares que foram encontradas em outras condições inflamatórias. Isso sugere que a natureza inflamatória da infecção por SARS-CoV-2 durante a gravidez pode causar eventos obstétricos e neonatais adversos. A exposição à inflamação intrauterina e alterações placentárias também pode resultar em defeitos multissistêmicos de longo prazo em bebês expostos (Prochaska et al., 2020).

O coronavírus não foi encontrado no leite materno até agora; portanto, pacientes puerperais infectados são recomendados para manter a lactação. Os testes realizados por Chen et al. (2020) para coronavírus no leite materno de nove mulheres foram todos negativos. Com base nos dados limitados coletados até o momento, a maioria dos pesquisadores recomenda a amamentação ou, pelo menos, a alimentação com mamadeira com leite materno ordenhado, como uma fonte potencial de anticorpos maternos benéficos para o recém-nascido; no entanto, como Rassmussen et al. (2020) enfatiza, anticorpos protetores para coronavírus não são, infelizmente, de longa duração. $\mathrm{O}$ bebê deve ser alimentado com mamadeira por uma pessoa saudável ou, com precaução, por sua mãe, que deve usar máscara facial e lavar as mãos com cuidado para evitar a transmissão. A Sociedade Internacional de Ultrassom em Obstetrícia e Ginecologia recomenda que em casos assintomáticos ou levemente afetados a amamentação e a colocação sejam consideráveis, sob a condição de uso de máscara facial materna e lavagem cuidadosa das mãos (Chen et al., 2020).

\section{Conclusão}

Os princípios gerais relativos ao manejo de Covid-10 durante a gravidez incluem isolamento precoce, procedimentos agressivos de controle de infecção, teste de SARS-CoV-2 e coinfecção, oxigenoterapia conforme necessário, prevenção de sobrecarga de fluidos, antibióticos empíricos (devido ao risco de infecção bacteriana secundária), monitoramento da contração fetal e uterina, ventilação mecânica precoce para insuficiência respiratória progressiva, planejamento individualizado do parto e abordagem baseada em equipe com consultas multiespecializadas.

O aumento da vulnerabilidade das mulheres grávidas bem como a experiência anterior de epidemias de coronavírus anteriores é motivo de preocupação para complicações maternas e fetais. O rápido surto da doença, combinado com a incerteza, como resultado direto da falta de dados científicos sólidos, tem forçado os obstetras a adaptarem suas práticas atuais de forma pragmática.

É importante estar vigilante quanto à disseminação da doença e ser capaz de fornecer uma implementação rápida de medidas de controle e gerenciamento de surtos assim que o vírus chegar à comunidade. Intervenções padrão para controlar qualquer infecção respiratória grave são a base do cuidado para qualquer mulher grávida com Covid-19 e devem ser implementadas agressivamente em um modelo de cuidado baseado em equipe. 


\section{Referências}

Chen, H., Guo, J., Wang, C., Luo, F., Yu, X., Zhang, W., Li, J., Zhao, D., Xu, D., Gong, Q., Liao, J., Yang, H., Hou, W., \& Zhang, Y. (2020). Clinical characteristics and intrauterine vertical transmission potential of COVID-19 infection in nine pregnant women: a retrospective review of medical records. Lancet (London, England), 395(10226), 809-815.

Collin, J., Byström, E., Carnahan, A., \& Ahrne, M. (2020). Public Health Agency of Sweden's Brief Report: Pregnant and postpartum women with severe acute respiratory syndrome coronavirus 2 infection in intensive care in Sweden. Acta obstetricia et gynecologica Scandinavica, 99(7), 819-822.

Dashraath, P., Wong, J., Lim, M., Lim, LM, Li, S., Biswas, A., Choolani, M., Mattar, C., \& Su, L. L. (2020). Doença por coronavírus 2019 (COVID-19) pandemia e gravidez. American Journal of Obstetrics and Gynecology, 222 (6), 521-531.

Dashraath, P., Nielsen-Saines, K., Madhi, S. A., \& Baud, D. (2020). COVID-19 vaccines and neglected pregnancy. Lancet (London, England), 396(10252), e22.

D'Souza, R., Ashraf, R., Rowe, H., Zipursky, J., Clarfield, L., Maxwell, C., Arzola, C., Lapinsky, S., Paquette, K., Murthy, S., Cheng, M. P., \& Malhamé, I. (2021). Pregnancy and COVID-19: pharmacologic considerations. Ultrasound in obstetrics \& gynecology: the official journal of the International Society of Ultrasound in Obstetrics and Gynecology, 57(2), 195-203.

Ellington, S., Strid, P., Tong, V. T., Woodworth, K., Galang, R. R., Zambrano, L. D., Nahabedian, J., Anderson, K., \& Gilboa, S. M. (2020). Characteristics of Women of Reproductive Age with Laboratory-Confirmed SARS-CoV-2 Infection by Pregnancy Status - United States, MMWR. Morbidity and mortality weekly report, 69(25), 769-775.

Galvão, C.M., Mendes, K.D.S., Silveira, R.C.C.P. (2010) Revisão integrativa: método de revisão para sintetizar as evidências disponíveis na literatura. In: Brevidelli MM, Sertório SCM. Trabalho de conclusão de curso: guia prático para docentes e alunos da área da saúde. 105-26.

Guan ,W., Ni, Z. \& Hu, Y (2020). Clinical Characteristics of coronavirus disease 2019 in China. N Engl J Med. 382: 1708-1720.

Huang, C., Wang, Y., Li, X., Ren, L., Zhao, J., Hu, Y., Zhang, L., Fan, G., Xu, J., Gu, X., Cheng, Z., Yu, T., Xia, J., Wei, Y., Wu, W., Xie, X., Yin, W., Li, H., Liu, M., Xiao, Y., Gao, H., Guo, L., Xie, J., Wang, G., Jiang, R., Gao, Z., Jin, G., Wang, J. \& Cao, B. (2020). Clinical features of patients infected with 2019 novel coronavirus in Wuhan, China. Lancet (London, England), 395(10223), 497-506.

Lapinsky, S. E. (2017). Management of acute respiratory failure in pregnancy. Semin Respir Crit Care Med. 38: 201-207.

Masmejan, S., Pomar, L., Lepigeon, K., Favre, G., Baud, D. \& Rieder, W. (2020). COVID-19 and pregnancy. Rev Med Suisse. 16(692):944-946.

Pacheco, L.D., Saade, G.R. \& Hankins, G.D.V. (2018). Extracorporeal membrane oxygenation (ECMO) during pregnancy and postpartum. Semin Perinatol. 42: $21-25$.

Prochaska, E., Jang, M., \& Burd, I. (2020). COVID-19 in pregnancy: Placental and neonatal involvement. American journal of reproductive immunology, 84(5), e13306.

Rassmussen, S. A., Smulian, J. C., Lednicky, J. A., Wen, T. S. \& Jamieson, D. J. (2020). Coronavirus disease (COVID-19) and pregnancy: what obstetricians need to know. Am J Obstet Gynecol. 222(5): 415-426.

Ruan, Q., Yang, K., Wang, W., Jiang, L., \& Song, J. (2020). Clinical predictors of mortality due to COVID-19 based on an analysis of data of 150 patients from Wuhan, China. Intensive care medicine, 46(5), 846-848.

Rajewska, A., Mikołajek-Bedner, W., Lebdowicz-Knul, J., Sokołowska, M., Kwiatkowski, S. \& Torbé, A. (2020). COVID-19 and pregnancy - where are we now? A review. J Perinat Med. 25;48(5):428-434.

Sutton, D., Fuchs, K., D'Alton, M., \& Goffman, D. (2020). Universal Screening for SARS-CoV-2 in Women Admitted for Delivery. The New England journal of medicine, 382(22), 2163-2164.

Thornton, J. G. (2020). COVID-19 in pregnancy. BJOG.127(9):1122.

Wan, S., Yi, Q., Fan, S., Lv, J., Zhang, X., Guo, L., Lang, C., Xiao, Q., Xiao, K., Yi, Z., Qiang, M., Xiang, J., Zhang, B. \& Chen, Y. (2020). Characteristics of lymphocyte subsets and cytokines in peripheral blood of 123 hospitalized patients with 2019 novel coronavirus pneumonia (NCP). medRxiv.

Wang, C. L., Liu, Y. Y., Wu, C. H., Wang, C. Y., Wang, C.H. \& Long, C. Y. (2021). Impact of COVID-19 on Pregnancy. Jornal internacional de ciências médicas, 18 (3), 763-767.

Wenling, Y., Junchao, Q., Xiao, Z., \& Ouyang, S. (2020). Pregnancy and COVID-19: management and challenges. Revista do Instituto de Medicina Tropical de Sao Paulo, 62, e62.

Yang, H., Wang, C. \& Poon, L. C. (2020). Novel coronavirus infection and pregnancy. Ultrasound Obstet Gynecol. 55:435-7. 